\title{
Sustainable Manufacturing: Application of Optimization to Textile Manufacturing Plants
}

By I.S.J.D. Liyanage, J.K.D. Sachira Nuwanga, Anjana W.P.G.R, Dr. W.H. Rankothge

\& Narmada Gamage

Sri Lanka Institute of Information Technology

Abstract- The main goal of manufacturing industry is to produce the end products on time with good quality and keep the resource wastage low. However, manufacturing industry face several challenges such as bottle necks in the workflow, unsynchronized production, and sudden increase in product demands.

In this paper, we are proposing a management platform for textile manufacturing plants with following modules: (1) sewing workflow optimization (2) quality assurance workflow optimization and (3) finishing workflow optimizations. We have used Genetic Programming (GP) approach, to optimize the workflows, considering different factors that affect each workflow. Our results show that, using our proposed platform, the manufacturing workflows can be optimized and reduce the bottle necks in the workflows and resource wastage in the manufacturing plant.

Keywords: manufacturing plant, textile industry, optimization, planning, scheduling, genetic programming (GP).

GJCST-H Classification: J.6

Strictly as per the compliance and regulations of:

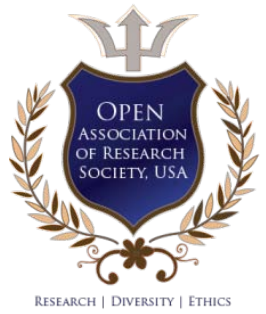

(C) 2020. I.S.J.D. Liyanage, J.K.D. Sachira Nuwanga, Anjana W.P.G.R, Dr. W.H. Rankothge \& Narmada Gamage. This is a research/review paper, distributed under the terms of the Creative Commons Attribution-Noncommercial 3.0 Unported License http://creativecommons.org/licenses/by-nc/3.0/), permitting all non-commercial use, distribution, and reproduction in any medium, provided the original work is properly cited. 


\title{
Sustainable Manufacturing: Application of Optimization to Textile Manufacturing Plants
}

\author{
I.S.J.D. Liyanage ${ }^{\alpha}$, J.K.D. Sachira Nuwanga ${ }^{\sigma}$, Anjana W.P.G.R ${ }^{\rho}$, Dr. W.H. Rankothge ${ }^{\omega}$ \\ \& Narmada Gamage $*$
}

Abstract- The main goal of manufacturing industry is to produce the end products on time with good quality and keep the resource wastage low. However, manufacturing industry face several challenges such as bottle necks in the workflow, unsynchronized production, and sudden increase in product demands.

In this paper, we are proposing a management platform for textile manufacturing plants with following modules: (1) sewing workflow optimization (2) quality assurance workflow optimization and (3) finishing workflow optimizations. We have used Genetic Programming (GP) approach, to optimize the workflows, considering different factors that affect each workflow. Our results show that, using our proposed platform, the manufacturing workflows can be optimized and reduce the bottle necks in the workflows and resource wastage in the manufacturing plant.

Keywords: manufacturing plant, textile industry, optimization, planning, scheduling, genetic programming $(\mathrm{GP})$.

\section{INTRODUCTION}

1 he textile industry is an important segment in the worlds' manufacturing industry, playing a crucial role in economic development. It is an evergrowing market, with key competitors being China, European Union, United States, and India [19]. The textile sector offers huge employment opportunities for people, especially for the people reside in rural area.

In the textile industry, specifically in manufacturing plants, the production process is a combined effort of different sections such as designing, cutting, sewing, packing, and delivering. They ensure that the clothes are produced with good quality and delivered on time with minimal wastage. However, the textile industry faces several challenges such as bottle necks in the workflow, unsynchronized production, sudden increases in product demands and unseen product wastages [20].

Therefore, it is necessary to adopt to a proper planning and scheduling approach, so that the workflows of manufacturing plants can be optimized. The optimal planning and scheduling are traditionally modeled as Integer Linear Programming (ILP)

Author $\alpha \sigma \rho \omega ¥$ : Sri Lanka institute of Information Technology, New Kandy Road, Malabe, Sri Lanka. e-mails: isuruliyanage1@gmail.com, ravinduanjana04@gmail.com, sachiranuwanga123@gmail.com, windhya.r@slit.lk,narmada.g@slit.lk optimization. However, there is the intrinsic constraint that ILP optimization is a NP-complete problem, and even when solutions are obtained for limited classes, it might be too slow [5]. Therefore, ILP calculation is unfeasible for optimization problems with dynamic nature, even though an ILP formalization of the problem gives an exact solution. The best approach would be to use an approximation approach (heuristic-based approach) for planning and scheduling of manufacturing plants activities.

In this paper, we have proposed a platform for planning and scheduling of a manufacturing plant, specifically targeting the textile industry. We have identified three main workflows of a textile manufacturing plants: sewing, quality assurance and finishing. Therefore, the proposed platform includes following modules: (1) sewing workflow optimization (2) quality assurance workflow optimization and (3) finishing workflow optimization. We have used an approximation (heuristic) based approach: Genetic Programming (GP), to optimize the identified work flows. The GP approach is a well-known method in the planning and scheduling context [1-16].Moreover, they have been widely used for research works related to manufacturing planning systems.

We have identified several parameters that effect each workflow, and processes required to be optimized in each workflow. We have consulted and visited textile manufacturing plants, their management and workers to identify the factors contributing to optimizing workflows. We have conducted experiments to measure the performances of our algorithms using the Sri Lankan textile manufacturing industry as a case study. With the real data sets, our results show that, our proposed GP approach can decide the optimal planning of each work flow dynamically in the order of seconds.

The rest of the paper is organized as follows. Section II presents the related work. Section III introduces the proposed optimization algorithm for planning and scheduling of textile manufacturing plants. In section IV, the result and discussions are presented. Our final remarks can be found on Section $V$. The references are mentioned in Section VI.

\section{Related WORK}

The Genetic Programming (GP) approach is a well-known method in the planning and scheduling 
context [1-16], especially for NP-hard problems, In this section, we will discuss the most recent work on scheduling and planning in the textile manufacturing context.

The work on [1] shows that resourceconstrained project scheduling problems are NP-hard and therefore, it is recommended to use approximation (heuristic) approaches to solve the problem. They have proposed a multi-objective evolutionary algorithm to solve the problem with multiple activity performance modes and two objectives to minimize project makes pan and resource utilization smoothness. They have used GP for their proposed algorithm. The authors of [2] used GP approach to implement an optimal resource allocation algorithm, that minimizes the average execution time of the critical path of workflow under constraints. The authors of [3] show that Master Production Scheduling (MPS) is the key action for success of a textile manufacturing plant. First, they have analyzed the constraints, mainly the installed capacity and the number of workers. Next, with the use of genetic algorithms, the MPS is optimized to carry out production planning, with an improvement of up to $96 \%$ of the level of service provided. The research [4] developed a system that uses GP approach to optimize pattern generation in textile production. Their results show that the system can generates enough patterns for fabric designers according to a given set of constraints. The work on [5] focuses on scheduling trousers collection orders, considering dates of starting orders and exporting them, the quantities of each order and the combined importance of customer and style. They have used GP approach to generate the best orders scheduling solution. The study of [6] addresses the production scheduling problem in a textile factory and they have used GP approach to find the minimum total make-span. The authors of [7] presented a methodology to design a control strategy to optimize a complex production process using a neural network combined with GP. The GP approach is used to optimize the architecture and the underlying parameters of the neural network in order to achieve the most effective model of the production process and to obtain set point values and raw material characteristics for an optimal tenacity and elongation of the spinning yarns.

Our research work has been inspired by these works, and we are focusing on a proposing a management platform which helps textile manufacturing plants to optimize their workflows and schedule them with minimum bottle necks and wastages.

\section{ili. Methodology}

We are proposing to use an approximation approach (heuristic-based approach) for planning and scheduling of textile manufacturing plants workflows, which have a dynamic nature. Specifically, we are proposing to use Genetic Programming (GP) approach. We have identified 3 workflows in the textile manufacturing plant to apply the optimization: sewing, quality assurance and finishing. We have implemented the E-Optimizer platform, which can be used to optimize sewing, quality assurance and finishing workflows. Figure 1 Shows the system diagram of E-Optimizer.

It is important to note that, there are several factors to be considered when optimizing selected workflows in the textile manufacturing plant. As the initial step, we have identified parameters that effect each workflow, and processes required to be optimized in each workflow. We have consulted and visited textile manufacturing plants, their management and workers to identify the factors contributing to optimizing workflows.

For sewing process, parameters such as number of pieces to be sew, number of pieces that can be handled by one machine, number of sewing machines and time taken for one item to be sewed, effect the effectiveness of workflow. In the quality assurance workflow, parameters such as availability of chemical stain removers, hot water and steam generators, number of washers, spinners, and dryers, effect the effectiveness of workflow. For the finishing workflow, parameters such as availability of hand irons with a vacuum press table, scissors press, carousel machines, steam dollies, availability of plastic bags, boxes, and cartons, effect the effectiveness of workflow.

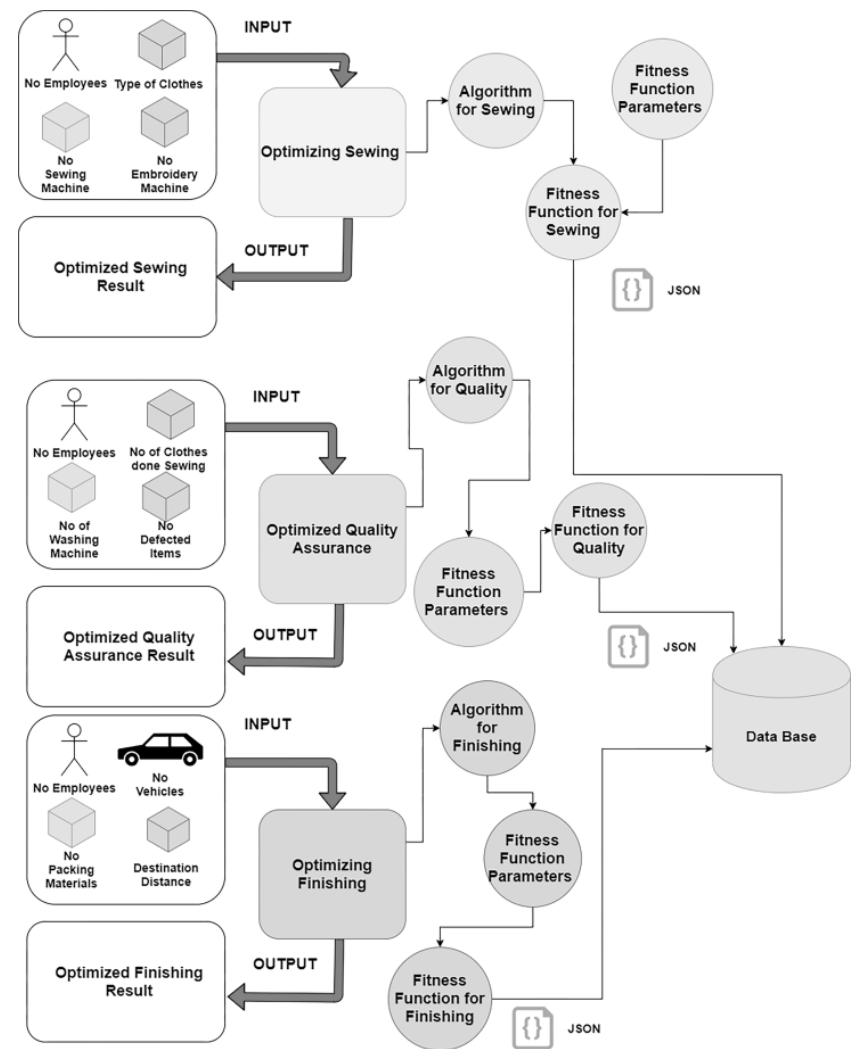

Fig. 1: System diagram of E-Optimizer 
a) Genetic Programming (GP) approach

GP is a method of processing the initial population (a group of individual solutions) to get adapted to a new better environment, through threemajor genetic operators: (1) selection, (2) crossover and (3) mutation [18]. The group of individual solutions gradually evolved into a better area of the search space by continuing to evolve from generation to generation. They will eventually converge to the most appropriate individual solution, which is the optimal solution for a problem.

GP can be described by the following five main steps [18]:

1. The initial population $P(0)$ is generated with $x$ solutions

2. The fitness value $V(p)$ is calculated for each individual solution $p$ in the current population $P(t)$

3. The next population $P(t+1)$ is generated, by selecting $n$ best solutions from $P(t)$

4. Offsprings are produced by applying the genetic operators to population $\mathrm{P}(\mathrm{t}+1)$

5. The process is repeat from Step 2 until a satisfying solution.

The process begins with generating a set of individuals which is called a Population. Each individual is a Solution to the problem. After the initial population is generated, afitness function is used to measure how good each solution is. Out of all the solutions, the best solutions are selected for next generation population.

Then genetic operations are applied to selected solutions to generate new solutions. We have considered two types of genetic operators to produce offspring: (1) mutation and (2) crossover. Crossover and mutation perform two different activities. Crossover is a convergence operation which is intended to pull the current population towards a local min/max. But the Mutation is a divergence operation, and it infrequently break one or more members of a population out of a local min/max space and possibly discover a better space. Since the end goal is to bring the population to convergence, crossover happen more frequently and mutation, being a divergence operation, happen less frequently.

The newly generated solutions are added to the existing set of solutions, which is known as the current population. This process is continued until y number of generations are explored. In the final generation, the solution that gives the best fitness value is selected as the best solution.

\section{b) Optimization of sewing workflow}

The optimization goal for sewing workflow is to optimize the sewing capacity arrangement; that is to find the best set of clothes to be sew within a given time limit. Cloth types are prioritized based on their profit generation capabilities, and given a priority, where high profit generating clothes are given a high priority value, to ensure maximum profit generation.

The sewing workflow optimization can be explained as the following constrained optimization: Maximize:

$$
\sum_{i=1}^{n} C_{i} \cdot P_{i}
$$

Subject to the constraint:

$$
\sum_{i=1}^{n} C_{i} \cdot A_{i} \leq X
$$

Where,

\begin{tabular}{|c|c|}
\hline $\mathrm{n}$ & Chromosome size \\
\hline $\mathrm{Ci}$ & $\mathrm{i}^{\text {th }}$ gene (Cloth type) \\
\hline $\mathrm{Pi}$ & Priority of the Cloth type $\mathrm{i}$ \\
\hline $\mathrm{Ai}$ & Time taken to sew the Cloth type i \\
\hline $\mathrm{X}$ & Time Threshold \\
\hline
\end{tabular}

The constraint (2) ensures that the selected set of clothes can be sewn within the given time threshold.

When we apply GP approach to the optimization problem, the encoding of a Chromosomes (optimal set of clothes to be sew within a given time limit) is demonstrated in Figure 2. The set of Chromosomes: A1, A2, A3 and A4 build the population. In a Chromosome, each gene supplies two pieces of information: (i) the gene index number represents the cloth type and (ii) the value in the gene signifies the whether the cloth type has been selected to sew or not. A " 1 "indicates that the respective item is selected and a " 0 " indicates that it is not selected. For example, the value of the last gene of Chromosome $\mathrm{A} 1$, indicates that the cloth type "Denim" has not been selected to sew.

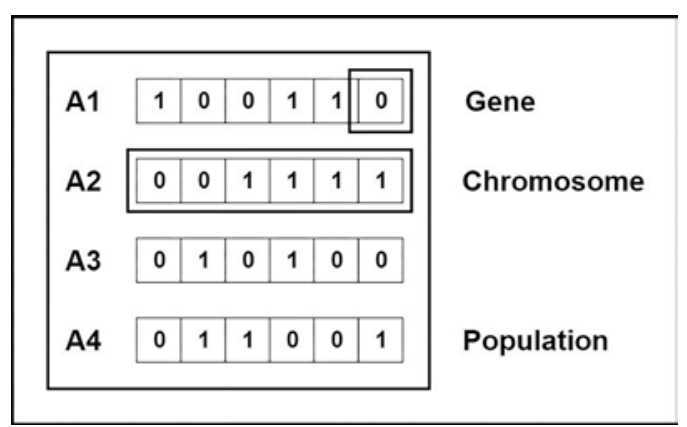

Fig. 2: Population built with Chromosomes A1, A2, $\mathrm{A} 3$ and $\mathrm{A} 4$

Each Chromosome is evaluated according to the fitness function given in Equation (3).

$$
\text { Fitness }=\sum_{i=1}^{n} C_{i} . P_{i}
$$

Next, the genetic operators are applied to Chromosomes to produce off-springs. Even though the traditional way of producing off-springs is to select the fittest chromosomes and perform genetic operations, it would lead to chromosomes that are closer to one another and results in less diversity. Therefore, we have used Roulette Wheel Selection method [17] and generated better off springs, with more diversity. 
In the Roulette Wheel Selection method, a circular wheel is divided into $n$ pies, where $n$ is the number of Chromosomes in the population. Each Chromosome gets a portion of the circle which is proportional to its fitness value.

As given in Table I, first, the fitness value percentage is calculated for each Chromosome.

Table 1: Fitness percentages of chromosomes

\begin{tabular}{|c|c|c|}
\hline Chromosome & Fitness & Percentage of Fitness \\
\hline A1 & 26 & 20.8 \\
\hline A2 & 25 & 20 \\
\hline A3 & 42 & 33.6 \\
\hline A4 & 32 & 20.6 \\
\hline
\end{tabular}

Then the Roulette Wheel is represented as shown in Figure 3, where each Chromosome gets a portion of the circle which is proportional to its fitness value.

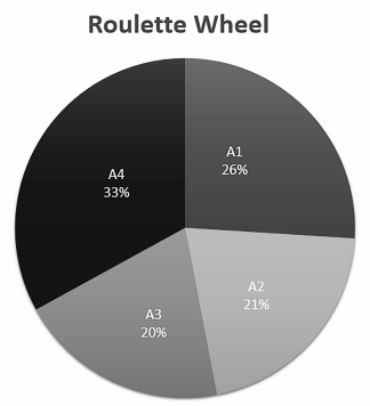

Fig. 3: Roulette Wheel Selection representation

To select parents to generate off-springs, first the wheel is rotated, and then the region of the wheel which comes in front of a fixed point is chosen as the parent. For the second parent, the same process is repeated. Genetic operations are performed to the selected two parents. We have performed one-point crossover and mutation to generate off-springs. This process is continued until $\mathrm{x}$ number of generations are explored. In thefinal generation, the solution that gives the best fitness value is selected as the best solution.

\section{c) Optimizaiton of quality assurance workflow}

The quality assurance process in textile industry ensures that there are no stains on manufactured clothes. Therefore, if a stain in spotted, the cloth is sent for cleaning. The optimization goal for quality assurance workflow is to optimize the cleaning process; that is to find the best set of clothes to be cleaned within a given time limit.

The quality assurance workflow optimization can be explained as the following constrained optimization:

Maximize:

$$
\sum_{i=1}^{n} C_{i} \cdot P_{i}
$$

Subject to the constraint:

$$
\sum_{i=1}^{n} C_{i} \cdot B_{i} \leq Y
$$

Where,

\begin{tabular}{|c|c|}
\hline $\mathrm{n}$ & Chromosome size \\
\hline $\mathrm{Ci}$ & $\mathrm{i}^{\text {th }}$ gene (Cloth type) \\
\hline $\mathrm{Pi}$ & Priority of the Cloth type $\mathrm{i}$ \\
\hline $\mathrm{Bi}$ & Time taken to clean the Cloth type $\mathrm{i}$ \\
\hline $\mathrm{Y}$ & Time Threshold \\
\hline
\end{tabular}

The constraint (4) ensures that the selected set of clothes can be cleaned within the given time threshold.

The GP approach used to optimize the quality assurance workflow is same as the GP approach used to optimize the sewing workflow, except for the fitness function, which measures how good a solution is.

The fitness function used in optimization of the quality assurance workflow is given in Equation (6).

$$
\text { Fitness }=\sum_{i=1}^{n} C_{i} \cdot P_{i}
$$

\section{d) Optimizing finishing workflow}

The finishing process in textile manufacturing carries out pressing and ironing of the cloth. The optimization goal for finishing workflow is to optimize the finishing process; that is to find the best set of clothes to be pressed and ironed within a given time limit.

The finishing workflow optimization can be explained as the following constrained optimization: Maximize:

$$
\sum_{i=1}^{n} C_{i} \cdot P_{i}
$$

Subject to the constraint:

$$
\sum_{i=1}^{n} C_{i} \cdot D_{i} \leq Z
$$

Where,

\begin{tabular}{|c|c|}
\hline $\mathrm{n}$ & Chromosome size \\
\hline $\mathrm{Ci}$ & $\mathrm{i}^{\text {th }}$ gene (Cloth type) \\
\hline $\mathrm{Pi}$ & Priority of the Cloth type $\mathrm{i}$ \\
\hline $\mathrm{Di}$ & Time taken to finish the Cloth type i \\
\hline $\mathrm{Z}$ & Time Threshold \\
\hline
\end{tabular}

The constraint (8) ensures that the selected set of clothes can be cleaned within the given time threshold.

The GP approach used to optimize the quality assurance workflow is same as the GP approach used to optimize the sewing workflow, except for the fitness function, which measures how good a solution is.

The fitness function used in optimization of the quality assurance workflow is given in Equation (9).

$$
\text { Fitness }=\sum_{i=1}^{n} C_{i} . P_{i}
$$

\section{Experiments and Results}

In this section, we are presenting the results and observations of the modules:(1) sewing optimization (2) quality assurance optimization and (3) finishing optimization, which were implemented using 
the GP based approaches. We have consulted and visited textile manufacturing plants, their management and workers to identify the factors contributing to optimizing workflows and to collect the data sets related to three workflows.

We have used GP as our heuristic-based optimization approach because it gives reasonable results fast. We derived three individual fitness functions for the three work flows, based on the factors effecting each flow. We have used mutation and one-point cross over as genetic operations and selected parents using Roulette Wheel approach to generate off-springs.

a) Optimizing sewing workflow

Case 1: As explained in the in previous section, with the GP process the given initial solution is improved by mutations and crossover operations over the generations. Therefore, we explored how GP process improves the initial solution when optimizing the sewing workflow. First, we find the initial solutions, and then apply GP process to improve the solution. As shown in the Figure 4, most of the improvements in the fitness function happens early on (during first 8 generations) and after that improvements happens very rarely.

Case 2: Next we explored the relationship between time and number of generations for sewing optimization process. As shown in Figure 5, time taken for generations shows a sub-linear growth, and the optimal values are produced within milliseconds.

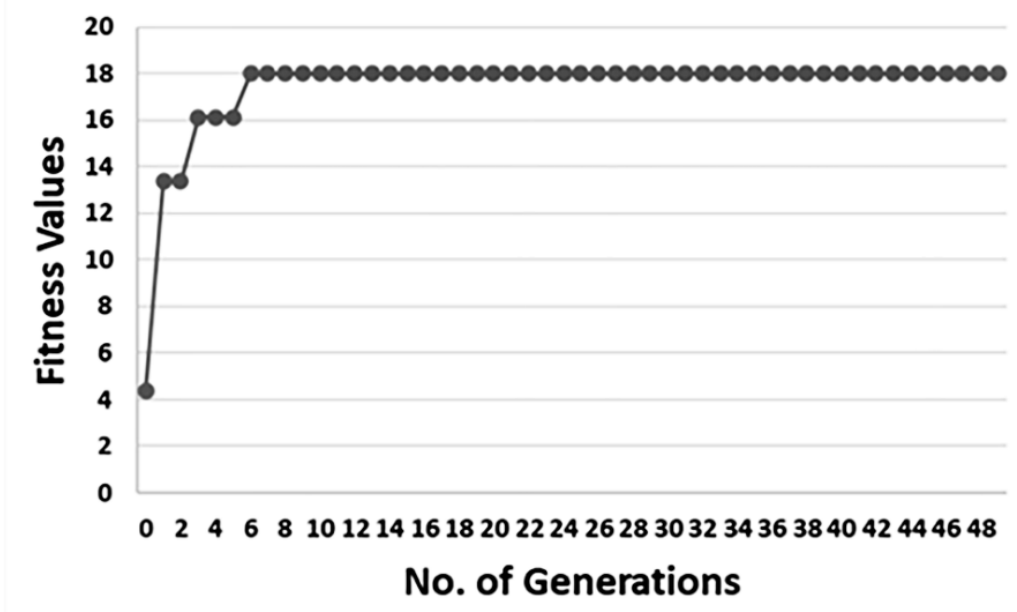

Fig. 4: Optimizing sewing: Effect of number of generations to Fitness Value

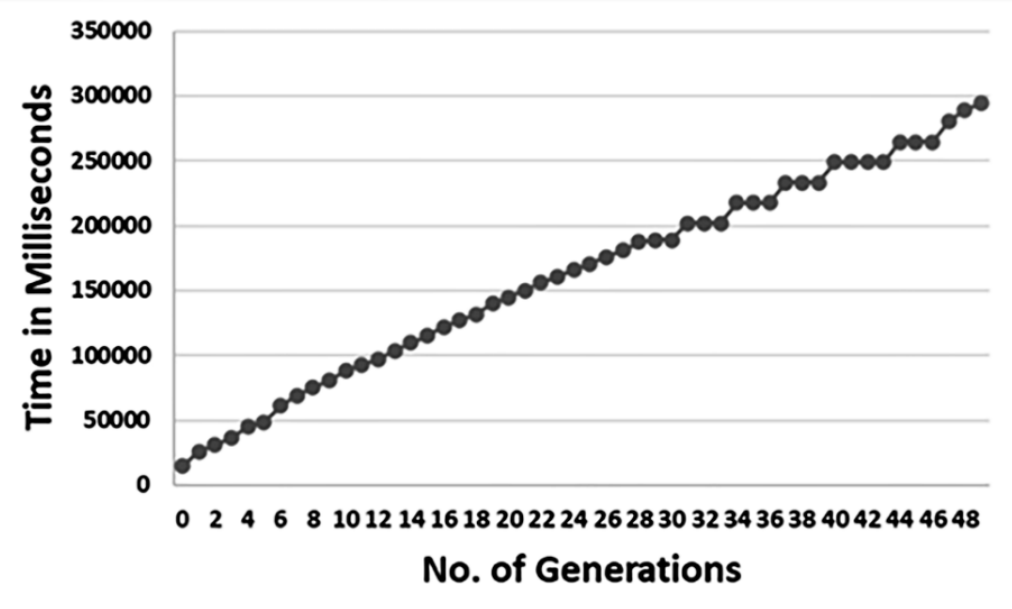

Fig. 5: Optimizing sewing: Effect of number of generations to Time

\section{b) Optimizing quality assurance workflow}

Case 3: To explore how the initial solution improves by applying GP process when optimizing the quality assurance workflow, we conducted experiments. First, we find the initial solutions, and then applies GP process to improve the solution. As shown in the figure 6, most of the improvements in the fitness function happens early on (during first 5 generations) and after that improvements happens very rarely. 


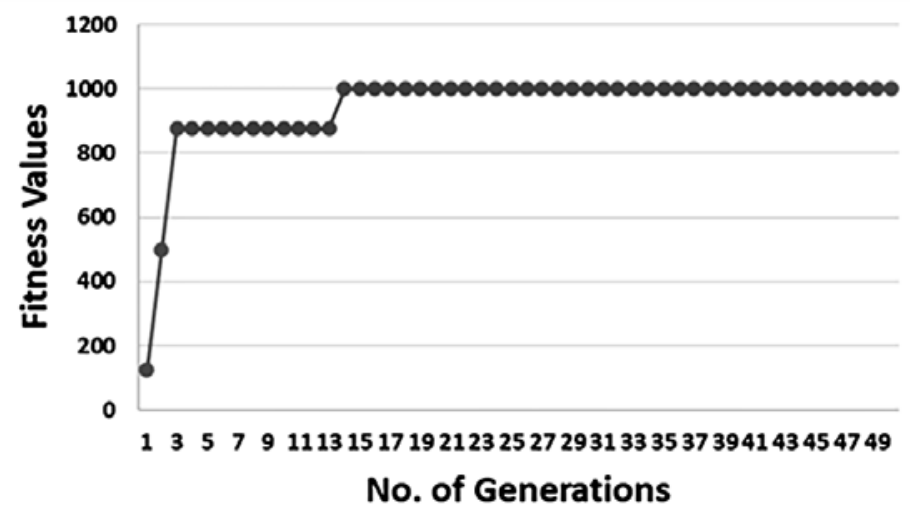

Fig. 6: Optimizing quality assurance: Effect of number of generations to Fitness Value

Case 4: We explored the relationship between time and number of generations for quality assurance optimization process. As shown in Figure 7, time taken for generations shows a sub-linear growth, and the optimal values are produced within milliseconds.

\section{c) Optimizing finishing workflow}

Case 5: To explore how the initial solution is improved with GP process when optimizing the finishing workflow, we conducted experiments. First, we find the initial solutions, and then applied GP process to improve the solution. As shown in the Figure 8 , most of the improvements in the fitness function happens early on (during first 6 generations) and after that improvements happens very rarely.

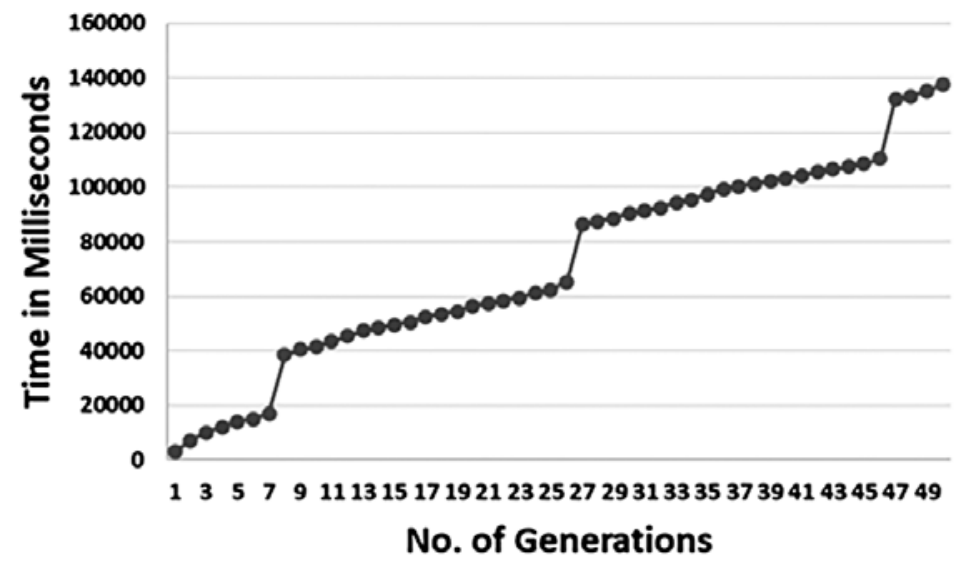

Fig. 7: Optimizing quality assurance: Effect of number of generations to Time

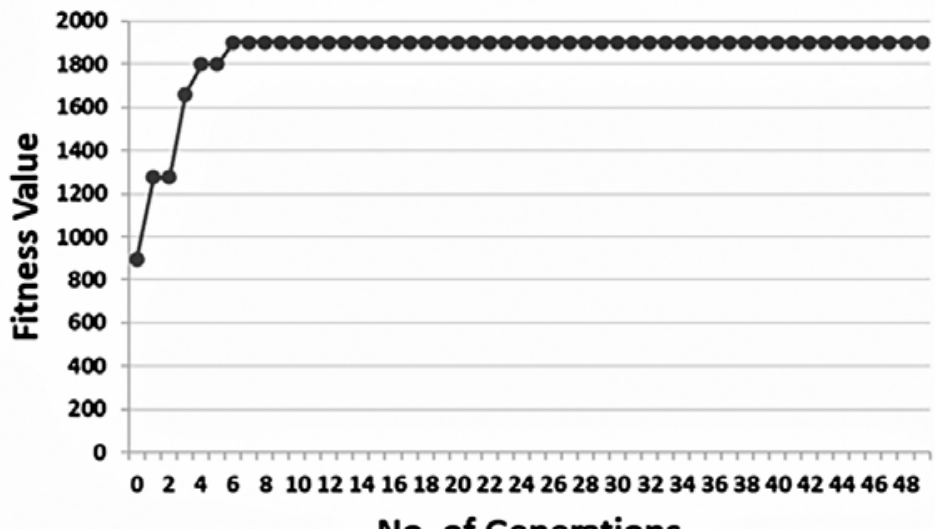

No. of Generations

Fig. 8: Optimizing finishing: Effect of number of generations to Fitness Value 
Case 6: Finally, we explored the relationship between time and number of generations for finishing optimization process. As shown in figure 9, time taken for generations shows a sub-linear growth, and the optimal values are produced within milliseconds.

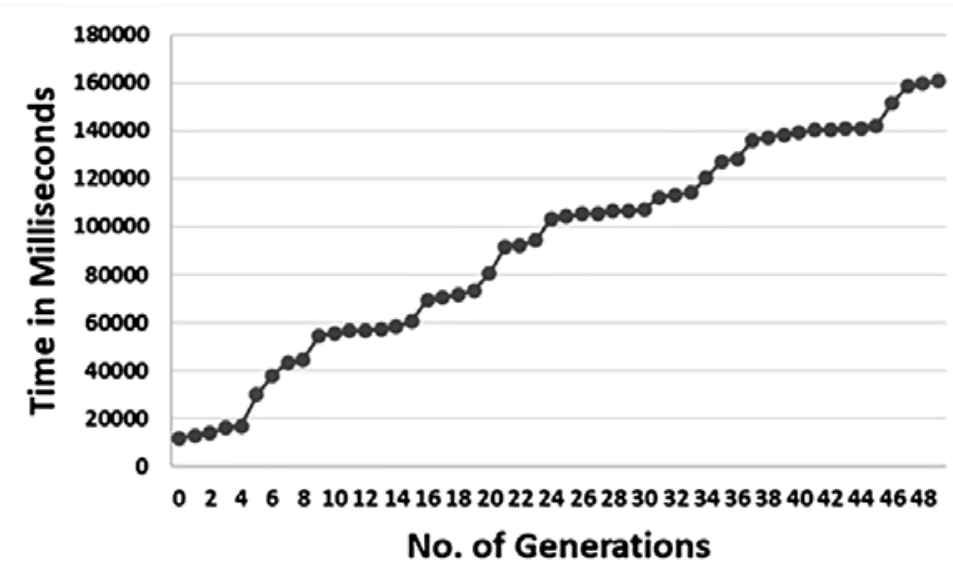

Fig. 9: Optimizing finishing: Effect of number of generations to Time

\section{Final Remarks}

We have proposed a management platform for optimization and scheduling of textile manufacturing plant, specifically for following workflows: (1) sewing optimization (2) quality assurance optimization and (3) finishing optimization. We have used Genetic Algorithms as our heuristic-based optimization approach to get reasonable results fast. We have consulted and visited textile manufacturing plants, their management and workers to identify the factors contributing to optimizing workflows. We derived three individual fitness functions for the three work flows, based on the factors effecting each flow. Our results show that, using our proposed modules, the manufacturing plant work flows can be optimized and scheduled efficiently and effectively.

As the future work, we are planning to extend the optimization research work, specifically to explore other heuristic approaches such as Iterated Local Search (ILS), and make recommendations on most suitable optimization approaches.

Also, we are planning to work on a prediction module, that predicts the expected time to finish workflow, specific for different cloth types, instead of using an average time threshold for all cloth types. The prediction module will help us to build a complete platform for optimization and scheduling of textile manufacturing plant.

\section{References Références Referencias}

1. S. Hartmann, "A competitive genetic algorithm for resource-constrained project scheduling," Naval Research Logistics, vol. 45, no. 7, pp. 733-750, 1998

2. Jisuanji Jicheng, Zhizao Xitong, "Optimization method of workflow resources allocation under cost constraints", 2007.
3. Leandro L. Lorente, Jefferson R. Murillo-Valle "Optimization of the Master Production Scheduling in a Textile Industry Using Genetic Algorithm", International Conference on Hybrid Artificial Intelligence Systems 2019.

4. Obe O., Egwuche O. S., "Genetic algorithm approach for fabric pattern generation in the textile industries", Annals. Computer Science Series, 2018.

5. Bessem Kordoghli, Seifeddine Saadallah, "Scheduling Optimization in a Cloth Manufacturing Factory Using Genetic Algorithm with Fuzzy Logic for Multi-Objective Decisions", Journal of Textile and Apparel, Technology and Management, 2010.

6. Harwin Kurniawan, Tanika D. Sofianti, "Optimizing Production Scheduling Using Genetic Algorithm in Textile Factory", Journal of System and Management Sciences 2014

7. S.Sette, L.Boullart, "Using Genetic Algorithms to Design an Overall Control Strategy of an Industrial Process", IFAC Proceedings Volumes, 1997

8. W. Rankothge, J. Ma, F. Le, "Towards making network function virtualization a cloud computing service", Proc. IEEE IM, pp. 89-97, 2015.

9. W. Rankothge, F. Le, A. Russo, J. Lobo," Optimizing Resource Allocation for Virtualized Network Functions in a Cloud Center Using Genetic Algorithms ", Proc. IEEE IM, pp. 89-97, 2015.

10. W. Rankothge, F. Le, A. Russo, J. Lobo," Experimental results on the use of genetic algorithms for scaling virtualized network functions", Proc. IEEE SDN/NFV, pp. 47-53, 2015.

11. M. R. S. Muthusinghe, Palliyaguru S. T., W. A. N. D. Weerakkody, A. M. Hashini Saranga, W. H. Rankothge, "Towards Smart Farming: Accurate Prediction of Paddy Harvest and Rice Demand", R10-HTC 2018. 
12. Dhanuka Perera, Chamod Rathnayaka, Sachin Dilan, Lasitha Siriweera, W. H. Rankothge, "Sustainable Tourism: Application of Optimization Algorithms to Schedule Tour Plans", R10-HTC 2018.

13. M.C. Jayasuriya, K.T. Galappaththi, D. Sampath, "Experimental Study on an Efficient Dengue Disease Management System: Planning and Optimizing Hospital Staff Allocation", International Journal of Advanced Computer Science and Applications 2018.

14. WDI Fernando, W.H. Rankothge, "Optimization of Customer-Friendly Manual Load Shedding System, ICAC 2019.

15. SMMM Senarath, MTK Perera, "Smart Platform for Film Shooting Management" CICT 2019.

16. C Thiranjaya, R Rushan, P Udayanga, "Towards a Smart City: Application of Optimization for a Smart Transportation Management System", ICIAfS 2018.

17. Roulette Wheel Selection https://www.tutorialspoint. com/genetic_algorithms/genetic_algorithms_parent selection.htm.

18. $\bar{M}$. Mitchell, An Introduction to Genetic Algorithms, Cambridge, MA, USA: MIT Press, 1998.

19. Kaushalesh Lal, "The Textiles and Clothing Industry and Economic Development: A Global Perspective", Innovation Policies and International Trade Rules.

20. Maeen Md. Khairul Akter, "Sustainability in Textile Industry; Reality and Challenges", Textile Focus, 2019. 